\title{
15 \\ The validity of Z-map model for mold and die machining
}

Park, J. W. and Choi, B. K.*

School of Mechanical Engineering, Yeungnam University

214-1 Dae-Dong, Kyoungsan

Kyoungbuk, 712-749 Korea

Tel: $+82-53-810-3524$,

Fax: +82-53-813-3703,

E-mail:jwpark@ynucc.yeungnam.ac.kr

* IE Dept., KAIST

373-1 Kusong-dong Yusong-gu

Taejon, 305-701 Korea

Tel: $+82-42-869-3115$,

Fax:+82-42-869-3110,

E-mail: bkchoi@bezier.kaist.ac.kr

\begin{abstract}
Z-map is a special form of discrete nonparametric representation in which the height values at grid points on the $x y$-plane are stored as a 2D array $z[i, j]$. While Z-map is the simplest form of representing sculptured surfaces and it is the most versatile scheme for modeling nonparametric objects, the application of Z-map to die machining (eg, finish cutting, clean-up cutting) aroused much controversy over its weaknesses: accuracy, singularities (e.g., vertical walls), and some excessive storage needs.

Although it has such limitations, much research on the application of Z-map can be found in various articles. However, research involving systematic analysis of Z-map to represent sculptured surfaces or CL (cutter location) surfaces for mold and die machining is rather rare.

Presented in this paper are the following: evaluation of the height value of a point which is not a pre-defined grid point, exact (within machining tolerance) Zmap representation of sculptured surfaces containing vertical walls and sharp edge lines (i.e., the common edges of adjoining faces which violate tangential
\end{abstract}


continuity) by adopting a Core Z-map or EZ-map (Extended Z-map) model, and several methodologies of computing CL surface in a Z-map form. Also presented are examples of die machining in industry.

\section{Keywords}

Surface modeling, Z-map model, CL Z-map, mold and die machining

\section{INTRODUCTION}

Surface modeling for CAD/CAM has been developed mainly based on parametric (polynomial) representations such as NURBS (Non-Uniform Rational $\mathrm{B}$-Spline surface). The parametric surface model is very powerful and flexible, but it also has some limitations or difficulties in handling certain geometrical problems (Choi 95).

In a nonparametric representation, a surface is defined by its height values at given points on the xy-plane, and it has the functional form

$$
z=f(x, y)
$$

when the surface is defined in Cartesian coordinates. Z-map is a special form of discrete nonparametric representation in which the height values at grid points on the $x y$-plane (domain) are stored as a 2D array $z[i, j]$ of the form (see figure 1)

$$
z[i, j] \text { with } x=x_{0}+d_{x}{ }^{*} i \text { and } y=y_{0}+d_{y}{ }^{*} j \text {. }
$$

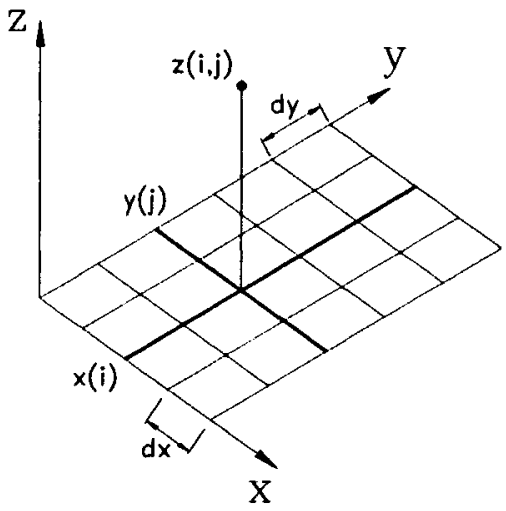

Figure 1 Z-map model

When a domain point $(x, y)$ is a grid point, the values of the index variables $i, j$ are determined from

$$
\mathrm{i}=\left(\mathrm{x}-\mathrm{x}_{\mathrm{o}}\right) / \mathrm{d}_{\mathrm{x}} \text { and } \mathrm{j}=\left(\mathrm{y}-\mathrm{y}_{\mathrm{o}}\right) / \mathrm{d}_{\mathrm{y}}
$$


and then its $z$-value is accessed from the array. If a point $(x, y)$ is not a grid point, its z-value can be estimated by some interpolating schemes.

The Z-map model has limitations of representing general 3-dimensional shapes including vertical walls, multi-leveled $z$-values (ie, more than one $z$-value is defined at a domain point), and sharp edges. For representing multi-leveled zvalues, a multi-level Z-map model which is known as dexel in the literature (Hook 1986, Kim 94) may be adopted.

Much research on Z-map applications can be found in various articles, including NC toolpaths verification (Andersen 78, Choi 94, Hsu 93, Kim 94, Takata 89), computer-aided process planning (CAPP) and die cavity machining (Choi 94). Also various research efforts in cutter path generation from the Z-map model (Choi 88, Takeuchi 89, Saito 91, Choi 93, Choi 94) can be found.

Section 2 shows a representation of vertical walls and sharp edges in a Z-map form by the use of some extended methods while retaining the simple (see figure 1) Z-map structure. Several methods to get an accurate (within a tolerance value) CL surface in a Z-map form are shown in the following section, and finally some examples are presented.

\section{Z-MAP REPRESENTATION OF SURFACE}

\subsection{Computation of a $z$-value at a pre-defined grid point}

Given a set of parametric surfaces, a Z-map $z[i, j]$ whose $x y$-domain bounds that of the surfaces can be obtained by evaluating the z-value(s) at each pre-defined grid point $[i, j]$ (see figure 2).

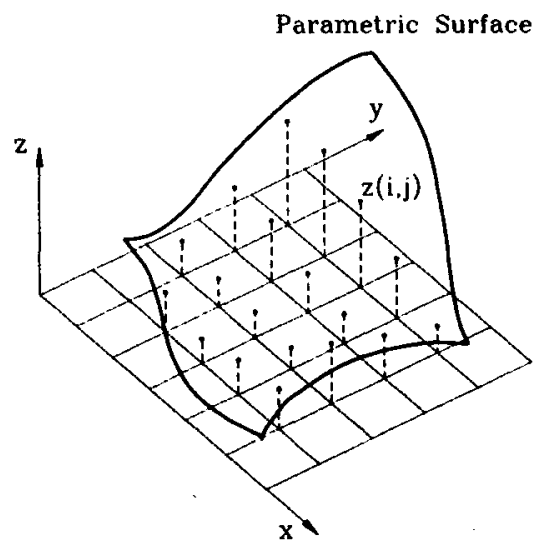

Figure 2 Pre-defined grid points and evaluation of z-values

Computing a $z$-value at an (x,y) point is equivalent to evaluating the 
intersection point $(x, y, z)$ between a surface and an infinite vertical line at $(x, y)$. This algorithm is called "2D Jacobian Inversion" (Choi 91), and efficiently computes the $z$-value within a given tolerance value. When multiple z-values are obtained, the highest one is chosen to avoid gouging.

Using differential geometry, the algorithm happens to fail to converge (ie, to evaluate an intersecting point). In that case, through a surface tessellation (e.g., triangulation) the $z$-values can be evaluated.

\subsection{Interpolation of a $\mathrm{z}$-value for a domain point}

As shown in the previous section, all the z-values of pre-defined grid points can be obtained, and they can be accessed directly from $z[i, j]$ by eqn. (3) as long as a domain point $(x, y)$ is a grid point.

The problem now becomes the evaluation of a $\mathrm{z}$-value at a domain point $(\mathrm{x}, \mathrm{y})$ which is not a pre-defined grid point. If a set of surfaces has neither a vertical wall nor a sharp edge, then we can assume without loss of generality that the overall surface is smooth enough to be well represented in a simple Z-map form such that the $z$-value at any arbitrary $(x, y)$ can be estimated through interpolating $z$-values of neighboring pre-defined grid points. Methods for handling vertical walls and sharp edges will be developed in the following section.

The authors use a "nonparametric cubic Bezier curve interpolating" scheme to estimate a z-value by considering $12 \mathrm{z}$-values of neighboring grid points. A nonparametric Bezier curve construction can be found in the literature (Choi 91, Farin 97).

Figure 3 shows 12 grid points on the xy-domain, which constitute four sets of control vertices $\left\{\mathrm{V}_{\mathrm{i}} ; i=0, . ., 3\right\}$ of nonparametric cubic Bezier curves (note that four grid points $\mathrm{gl}, \mathrm{g} 2, \mathrm{~g} 5, \mathrm{~g} 6$ are used twice) such that

$\left\{\mathrm{V}_{\mathrm{i}}\right\}_{1}=\{\mathrm{g} 0, \mathrm{gl}, \mathrm{g} 2, \mathrm{~g} 3\}, \quad\left\{\mathrm{V}_{\mathrm{i}}\right\}_{2}=\{\mathrm{g} 4, \mathrm{~g} 5, \mathrm{~g} 6, \mathrm{~g} 7\}$,

$\left\{V_{i}\right\}_{3}=\{\mathrm{g} 9, \mathrm{~g} 5, \mathrm{gl}, \mathrm{g8}\},\left\{\mathrm{V}_{\mathrm{i}}\right\}_{4}=\{\mathrm{gl} 1 \mathrm{l}, \mathrm{g} 6, \mathrm{~g} 2, \mathrm{~g} 10\}$.
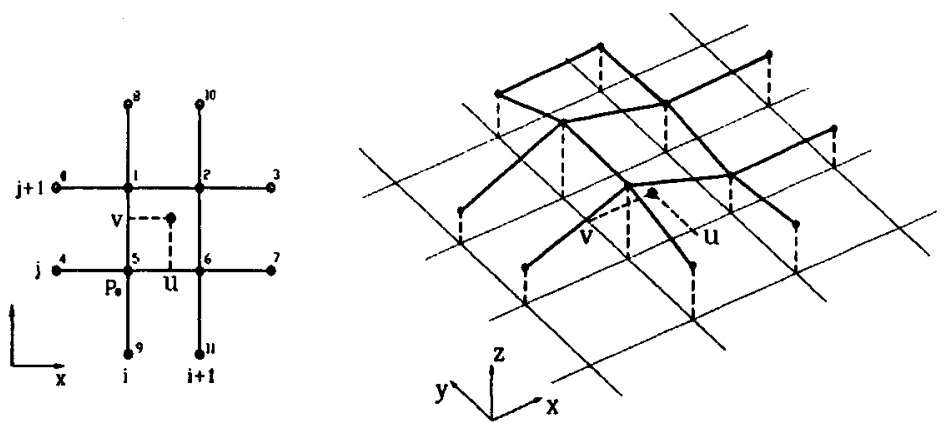

Figure 3 Nonparametric Bezier interpolation

Then each $\left\{V_{i}\right\}$ makes a nonparametric Bezier curve, and four z-values can be 
evaluated from each curve equation with parameter domain values of $u$ or $v$ (ie, $u$ for 2 horizontal curves, $v$ for 2 vertical ones, respectively). The $(u, v)$ pair is determined by considering local coordinate values of $(x, y)$ within the local rectangular domain $(\mathrm{gl}, \mathrm{g} 2, \mathrm{~g} 5, \mathrm{~g} 6)$ with $(\mathrm{u}, \mathrm{v})=(0,0)$ at Po. Now the $\mathrm{z}$-value at $(\mathrm{u}, \mathrm{v})$ can be estimated through a linear blending of the four $z$-values. If the domain point $(x, y)$ falls into a grid point $[i, j]$ within a tolerance, then $z[i, j]$ becomes the estimated z-value.

For a domain point $(x, y)$ where all 12 neighboring grid points cannot be defined (e.g., on the xy-domain boundary), linearly extended dummy grid points are added to construct a complete set of control vertices.

Comparing the estimated z-values with the exact ones on two typical smooth surfaces, we got the following results ( $\varepsilon=$ =error value):

$\varepsilon_{\max }= \pm 1.2 \times 10^{-4}(\square)$ for a bilinear surface: $\mathrm{z}=100 \sin (\pi \times / 180)$,

$\varepsilon_{\max }= \pm 3.0 \times 10^{-5}(\square)$ for an upper hemisphere: $x^{2}+y^{2}+z^{2}=100^{2}, z>70$,

larger $\varepsilon$-values occur on near-boundary areas (as predicted),

with a grid step distance (interval) of $d_{x}=d_{y}=1 \square$ (usually $0.7 \square$ for a real application). Considering that a typical machining tolerance value in finish-cut machining of mold and die is in the range of $10^{-3}$ to $10^{-2} \mathrm{\square}$, we can say that the simple Z-map representation works fine for practical mold $\&$ die machining as long as the overall surface is smooth. Figure 4 shows the scaled error patterns for those sample surfaces with $d_{x}=d_{y}=10 \square$ and $d_{x}=d_{y}=20 \square$, respectively.

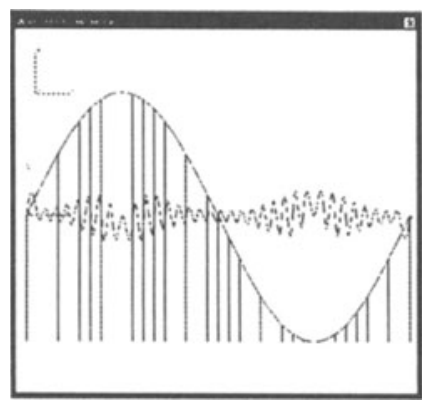

(a) $z=100 \sin (\pi x / 180)$

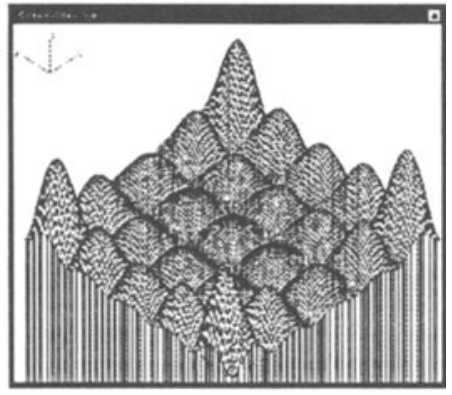

(b) $x^{2}+y^{2}+z^{2}=100^{2}, z>70$

Figure 4 Scaled error patterns when (a) $d_{x}=d_{y}=100$, (b) $d_{x}=d_{y}=20 \square$

\subsection{Extended models for handling vertical walls and sharp edges}

Most mold die-surfaces contain such features as sharp edge-lines and vertical walls, and so does automobile press die-surfaces (e.g., sharp convex edge-lines on an outer hood surface). The simple Z-map model, however, has the power to represent the special features only with a very small grid interval value (see figure 5), which cannot always be applied to finish machining due to excessive memory storage needs. Accordingly, some other methods should be conceived to overcome such difficulties. 

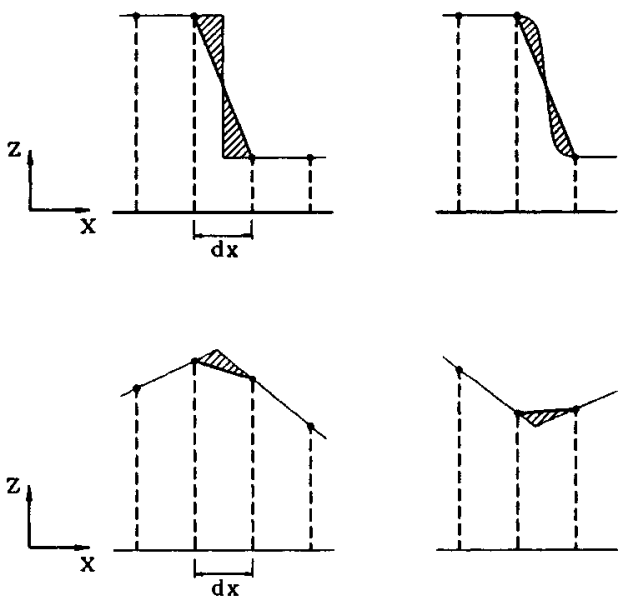

Figure 5 Wrongly defined vertical walls and sharp edges in a simple Z-map form

In order to represent the local features accurately (within a tolerance), the authors adopted the following auxiliary models:

- Core Z-map model,

- EZ-map (Extended Z-map) model.

- Core Z-map model

A core Z-map, with a much smaller $d_{x}$ and $d_{y}$ (equal to machining tolerance in a real application) than that of a simple Z-map, can be thought as a hard steel core embedded selectively (and locally) into a large die-surface area. In other words, it locally enhances the accuracy of the original Z-map on which it is located (see figure 6). Owing to selective localization much less memory storage is required, which, while preserving the accuracy and the Z-map form, makes it feasible to handle a large domain.

The "selective" locating of core maps is made possible by identifying areas of vertical wall and sharp edge lines (e.g., analyzing the Z-map and parametric surfaces). Then, a series of local domains of core Z-maps are defined and constructed along a sharp edge line or a vertical wall line (a series of domain points each with a local maximum gradient), as shown in figure 6 . In general, different grid interval values for $d_{x}, d_{y}$ of a core $Z$-map are assigned to minimize the memory usage. Evaluating a $\mathrm{z}$-value on a core map (when a domain point $(x, y)$ falls within the core map's domain) is similar to that on a Z-map (section 2.2). 

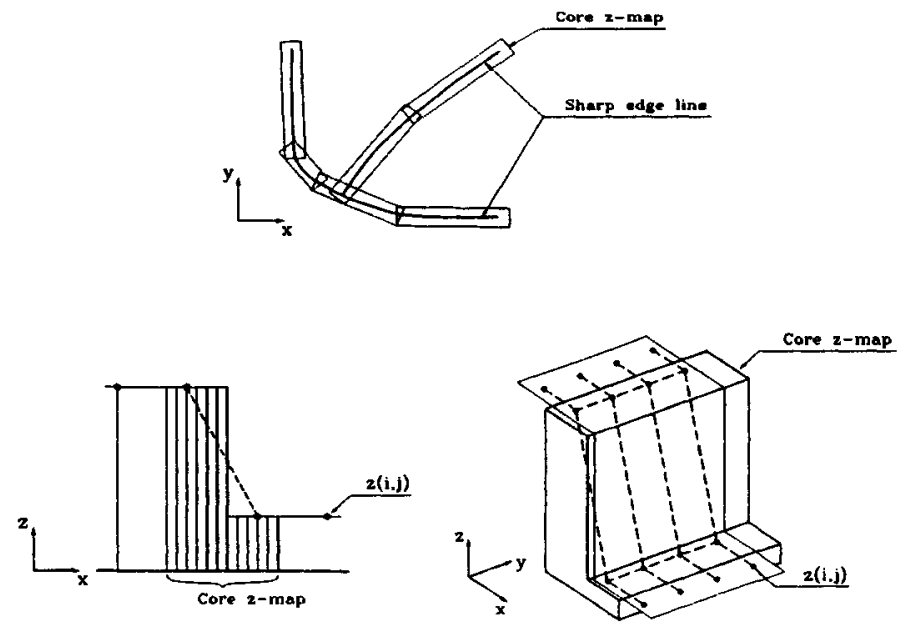

Figure 6 Schematic model of Core Z-map

\section{- EZ-map model}

The EZ-map (Extended Z-map) model defines "edge grid points" on each selected grid-line of a Z-map to recover the accuracy, while preserving the "localization" property (see figure 7). A grid-line of a Z-map means a 3D line segment connecting two consecutive 3D grid points. Identifying a grid-line on which edge grid points should be added is similar to the core Z-map model, and the number of points is determined to satisfy the machining tolerance $\delta$ (i.e., a function of $d x, d y, \delta)$.
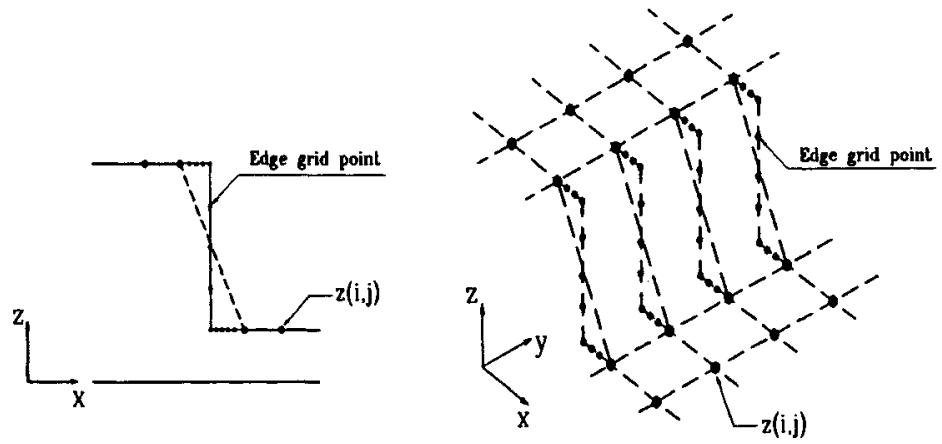

Figure 7 Schematic model of EZ-map

Evaluating the $\mathrm{z}$-value at an $(\mathrm{x}, \mathrm{y})$ domain point is similar to a Z-map, except that the edge grid points (if any) are additionally utilized and sharp edge lines can be considered. 


\section{Z-MAP REPRESENTATION OF CL-SURFACE}

\subsection{CL surface and the singularity}

A parametric surface model can be represented as a vector valued function

$$
r(u, v)=(x(u, v), y(u, v), z(u, v))
$$

and it is in fact a regular mapping from the parameter domain (u,v) to the 3D cartesian space $(x, y, z)$. The CL surface of $r(u, v)$ for a general round-end milling cutter with a fixed cutter axis can be defined such that

$$
\begin{aligned}
& \mathbf{r}^{0}(\mathrm{u}, \mathrm{v})=\mathbf{r}(\mathrm{u}, \mathrm{v})+\alpha(\mathbf{n}-\mathbf{u})+(\mathrm{R}-\alpha)(\mathbf{n}-\lambda \mathbf{u}) /\left(1-\lambda^{2}\right)^{1 / 2}(5) \\
& \text { where } \lambda=\mathbf{n} \cdot \mathbf{u}, \\
& \mathbf{n}=\mathbf{n}(u, v): \text { unit normal vector at }(u, v), \\
& \mathbf{u}=(0,0,1): \text { cutter axis, } \\
& \mathrm{R}: \text { cutter radius (half the diameter }), \\
& \\
& \alpha \cdot \text { cutter nose radius, }
\end{aligned}
$$

and any CL point of the cutter (i.e., reference point) is located on $\mathbf{r}^{\circ}(u, v)$. Then, eqn. (5) can be reduced to CL surfaces for ball or flat-end milling cutters if $\alpha$ becomes $\mathrm{R}$ or zero, respectively. The CL surface $\mathbf{r}^{\circ}(u, v)$ is also a vector-valued function $(x(u, v), y(u, v), z(u, v))$, and it may be a regular mapping from $(u, v)$ to $(\mathrm{x}, \mathrm{y}, \mathrm{z})$.
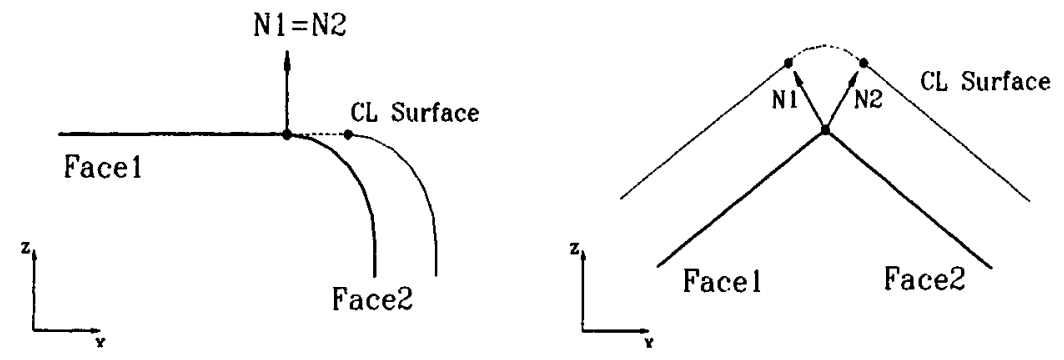

(a) For flat-end cutter

(b) For all cutter types

Figure 8 Singularity of a CL surface

There possibly exists, however, a CC (cutter contact) point $\mathbf{r}(\mathrm{u}, \mathrm{v})$ at which a regular mapping is impossible due to a singularity (see figure 8), and a unique CL point cannot be determined. For example, figure 8-b shows a sectional view of two adjoining faces and a common edge, which is convex and tangentially discontinuous. In the case of a ball-end milling cutter, a point on the edge curve should be mapped into a curve (e.g., a circular arc for a ball-end milling cutter) rather than a point, and the edge curve into a surface (eg, a cylindrical surface for a 
ball-end milling cutter) to fill the gap on the CL surface (dotted line). Figure 8-a shows another type of singularity at which, for a round or flat-end milling cutter, a gap (dotted line for a flat end-milling cutter ) on the CL surface is inevitable without special treatment.

Some methods to construct a gouge-free CL surface are presented in the following sections.

\subsection{CL surface construction}

The computation of a CL surface has been a very important issue in cutter path generation, which should be accurate (i.e., avoiding overcut and undercut) and efficient in its computation time. A CL surface construction (i.e., offsetting) based on the simple Z-map model can be found in the literature (Saito 91, Takeuchi 89). However, hardly any enhancement in accuracy has been demonstrated.

The authors' research group developed the following algorithms to obtain an accurate (within machining tolerance) CL Z-map (EZ-map):

- Z-map-based inverse offset,

- Surface-based inverse offset,

- Surface-based offset-facet sampling \& edge-based inverse offset,

where "surface" means a parametric surface, and "Z-map" can be easily extended to an EZ-map form.

\section{- Z-map-based inverse offset}

A Z-map obtained from parametric surfaces can be considered as a soft "master model", with all the grid points and edge grid points (if any) containing exact zvalues. By applying the inverse offset method of a Z-map (Choi 91) to generate a CL surface, we can obtain a CL Z-map directly from the master model Z-map. Figure 9 shows the schematic view of this method for a ball-end milling cutter.
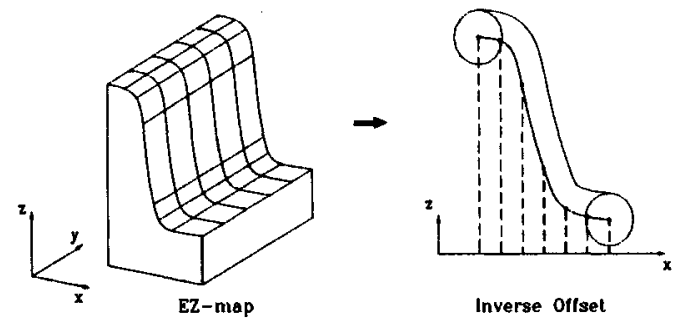

Figure 9 Z-map-based inverse offset (ball-end milling case)

The main advantages of the method are speed (i.e., efficiency in computation time) and robustness, that is, in any case it is possible to obtain a CL Z-map 
regardless of the singularity problem.

\section{- Surface-based inverse offset}

While it is a Z-map that the previous method employs as a master model, this method utilizes the surface itself; sampled isoparametric curves and all the boundary edge curves (see figure 10). Each curve consists of a series of 3D points, and grid points on the xy-domain are updated so that a CL Z-map can be constructed by the inverse offset of 3D points sequence (i.e., inverse cutting simulation along the 3D points). The 3D step-over distance between consecutive isoparametric curves of a surface is determined from differential geometry so that it satisfies a given tolerance value. The singularity problem (figure 8) is resolved through the inverse offset of all boundary edge curves.

It takes much more computation time than the previous method, since the inverse offset should be applied to closely positioned 3D points from the curves. In addition, most points in the previous method are regularly spaced grid points (on the xy-domain), which makes it possible to utilize a more efficient optimizing process (i.e., minimizing the updating area on the domain).

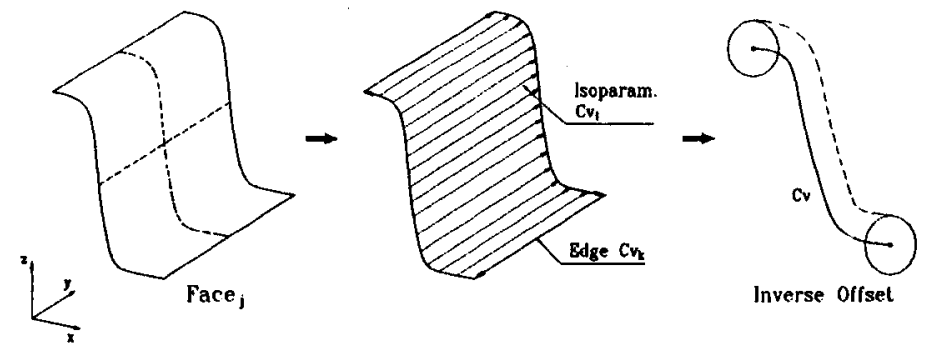

Figure 10 Surface-based inverse offset (ball-end milling case)

\section{- Surface-based offset-facet sampling \& edge-based inverse offset}

This can be said to be the most efficient method among the three, considering both accuracy and speed. We construct a CL Z-map by evaluating the 3D "offset triangles" of surfaces rather than doing the inverse offset of isoparametric curves. The triangular facet model of a surface can be obtained through tessellation of the surface, and each triangle is represented by three vertices located on the surface. Then, the "offset triangles" of a surface are constructed so that all vertices on the surface are moved to the corresponding $\mathrm{CL}$ points according to a given cutter type (Eqn.(5)) to become "offset vertices".

The same inverse offset algorithm is applied to all the boundary edge curves to resolve the singularity problems, as in the previous method (see figure 11). 

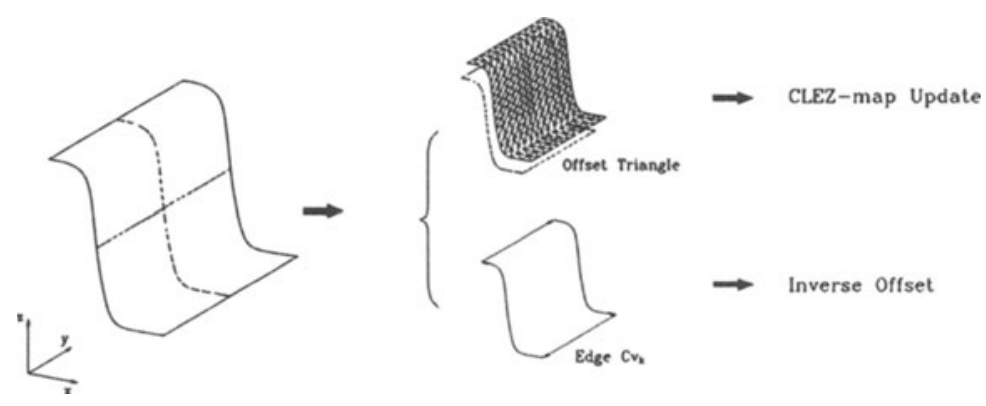

Figure 11 Surface-based offset-facet sampling and edge-based inverse offset

Finally, some error types are defined as follows:

- $\varepsilon_{1}=$ Z-map intrinsic error for representing CL surface $\left(\mathbf{r}^{\circ}(u, v)\right)$,

- $\varepsilon_{2}=$ Z-map intrinsic error for representing CC surface $(r(u, v))$,

- $\varepsilon_{3}=$ Discretization error of isoparametric curves,

- $\varepsilon_{4}=$ Triangulation error.

The second error value $\left(\varepsilon_{2}\right)$ comes from the difference between parametric surfaces and the "master model" Z-map, the first $\left(\varepsilon_{1}\right)$ from that between a CL Z-map and the ideal CL surface. The third one $\left(\varepsilon_{3}\right)$ is actually a combination of the following two; error in discrete 3D points of an isoparametric curve on the given surface, and error caused by non-zero step-over distance between the isoparametric curves. The error in discrete 3D points of a boundary edge is neglected. The triangulation error $\left(\varepsilon_{4}\right)$ comes from the difference between the ideal CL surface and an offset triangulated facet model. Roughly speaking, the relations between errors are; $\varepsilon_{1}=\varepsilon_{2}>\varepsilon_{3}>\varepsilon_{4}$. Now, Table 1 summarizes the presented methods.

Table 1 Summary: Z-map representation of CL surface

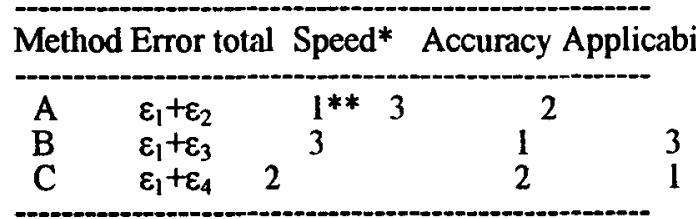

A. Z-map-based inverse offset,

B. Surface-based inverse offset,

C. Surface-based offset-facet sampling \& edge-based inverse offset.

* Speed for construction of CL surface.

**Number denotes rank (the larger the worse)

\section{EXAMPLES}

Figure 12 shows construction of a "master model" Z-map, its CL Z-map, and parallel-to-plane toolpaths for a very simple model surface (part of a connecting rod). The xy-domain of the Z-map is $110 \times 120 \mathrm{D}$, and the grid interval is $0.7 \square$. 
When the Z-map is represented in an EZ-map form with tolerance $=0.02$, about $400 \mathrm{Kbytes}$ memory storage is needed (about $7 \%$ of all grid lines contain edge grid points, with 40 points each). So for the CL Z-map, roughly.

Figure 13 shows another example with 1880 faces total. The xy-domain is $1200 \times 9000$ with a grid interval of 10 . The EZ-map requires about 10Mbytes memory storage, given the tolerance $=0.02$. In this case, about $3 \%$ of $Z$-map grid lines have edge grid points (with 50 points each).
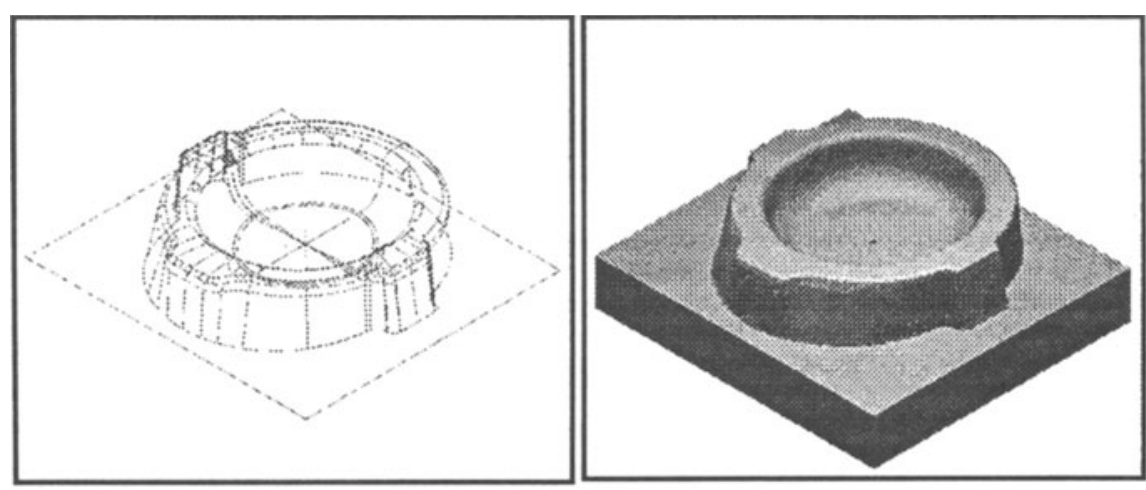

(a) $\mathrm{CAD}$ data

(b) Z-map model
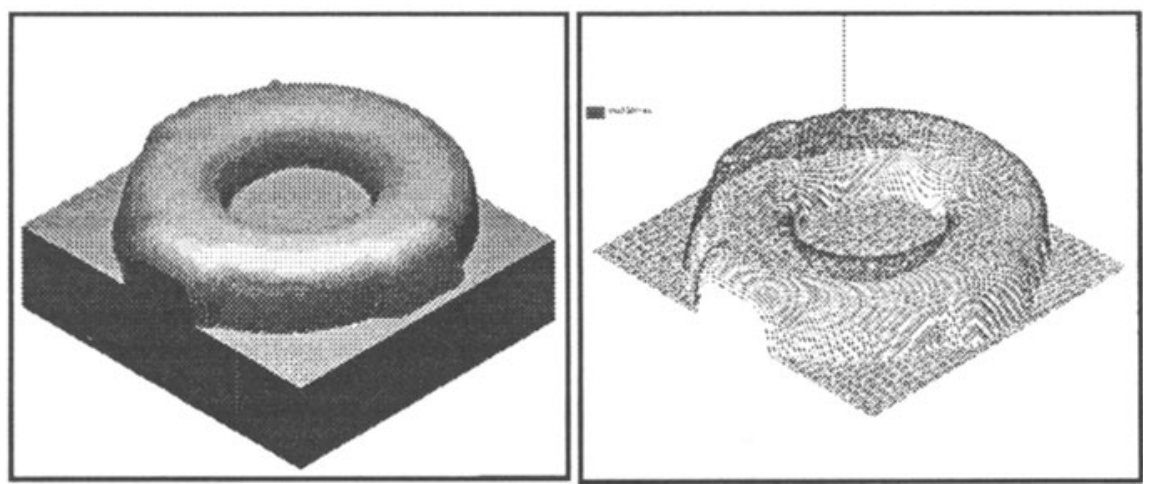

(c) CL Z-map

(d) Parallel-to-plane toolpaths

Figure 12 Application example: connecting rod (partial) 


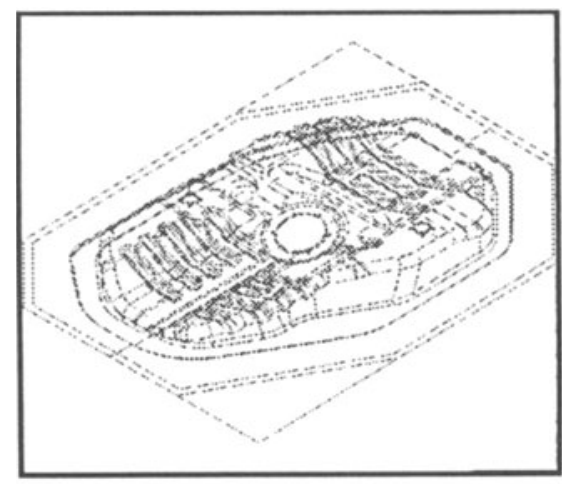

(a) CAD data

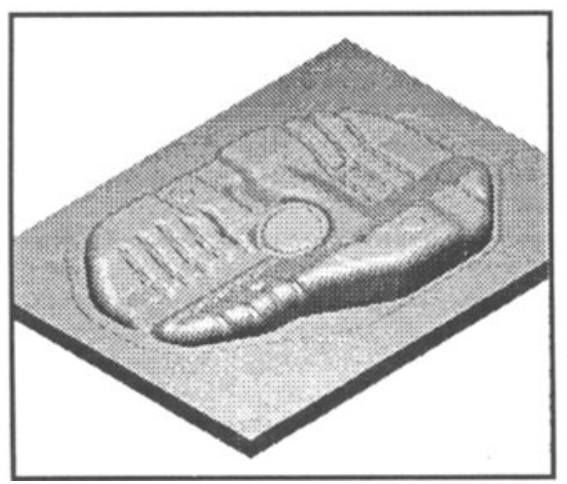

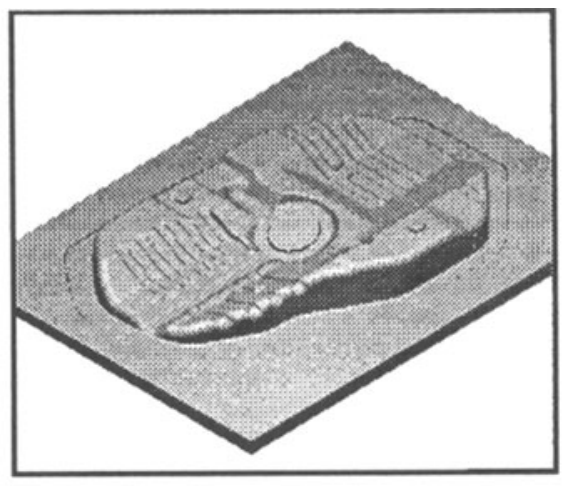

(b) Z-map model

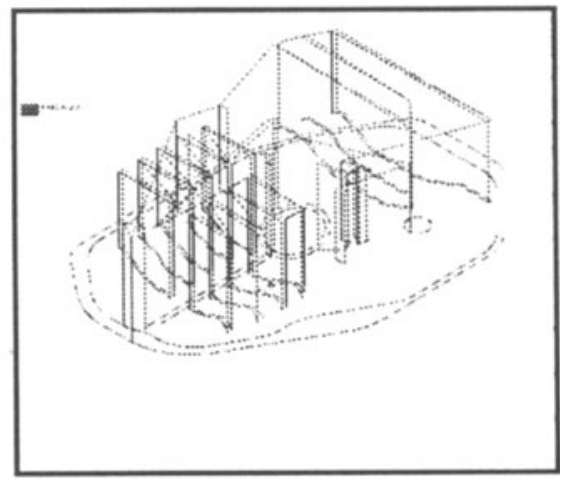

(d) Pencil curve machining toolpaths

(c) CL Z-map

Figure 13 Application example: fuel tank

\section{CONCLUSIONS}

It has been shown that the Z-map model and its extended models are powerful tools that can be successfully utilized in manufacturing. The main limitations of Zmap are the resolution problem in dealing with "vertical walls" and "real" sharp edges and the $\mathrm{O}(\mathrm{n})$ complexity problem (i.e., the memory and time complexity is proportional to the square of the resolution). Two approaches to handling these problems were illustrated; namely, core Z-map and EZ-map models. In addition, three methods for constructing a CL surface in a Z-map (or EZ-map) form were presented.

In fact, Z-map type models are utilized in many of the leading commercial CAM software systems (Cliks 91, Worknc 93), and in a few in-house systems for Chrysler Corporation (METD, MI, USA), Hyundai Motor Company (Korea), and 
other mold \& die manufacturing companies. The commercial CAM software ZMASTER (Zmaster 92) employed a Z-map type model equipped with the presented algorithms, and has been used for mold \& die manufacturing.

\section{REFERENCES}

Andersen,R.O.(1978) Detecting and eliminating collisions in NC machining. Computer-Aided Design, V10 N4, 231-237.

Choi,B.K.(1991) Surface modeling for CAD/CAM. Elsevier, New York.

Choi,B.K., Park,J.W. and Jun,C.S.(1993) Cutter-location data optimization in 5axis surface machining. Computer-Aided Design, V25 N6, 377-386.

Choi,B.K., Chung,Y.C., Park,J.W. and Kim,D.H.(1994) Unified CAM-system architecture for die and mould machining, Computer-Aided Design, V26 N3, 235-243.

Choi,B.K., Chung,Y.C. and Park,J.W.(1995) Application and extension of Z-map model, Proc. of the Pacific Conference on Computer Graphics and Applications, Seoul, Aug, 221-234.

Cliks(1991) CLIKS user manual. ARGO Technos 21, Japan.

Farin,G.(1997) CAGD: a practical guide. Academic Press, New York.

Hook,T.V.(1986) Real time shaded NC milling display. Computer Graphics, V20 N4, 15-20.

Hsu,P.L. and Yang,W.T.(1993) Realtime 3D simulation of 3-axis milling using isometric projection. Computer-Aided Design, V25 N4, 215-224.

Kim,C.B. and Yang,M.Y.(1994) A study on the verification of 5-axis CNC machining. Journal of KSME, V18 NI, 93-100 (in Korean).

Saito,T. and Takahashi,T.(1991) NC machining with G-buffer method. Computer Graphics, V25 N4, 207-216.

Takata,S. Tsai,M.D. and Inui,M.(1989) A cutting simulation system for machinability evaluation using a workpiece model. Annals of the CIRP, V38, 417-420.

Takeuchi,Y. Sakamoto,M. Abe,Y. and Orita,R.(1989) Development of a personal $\mathrm{CAD} / \mathrm{CAM}$ system for mold manufacture based on solid modeling techniques. Annals of the CIRP, V40, 455-458.

Worknc(1993) WORKNC user guide version 3.5. Sescoi, France.

Zmaster(1992) Z-Master reference manual. Cubic Tech, Korea.

\section{BIOGRAPHY}

Jung W. Park is a full-time lecturer of mechanical engineering. He received a BS in physics from Seoul National University, Korea, in 1987, and an MS from the Korea Advanced Institute of Science and Technology in 1991, also a Ph.D. in 1995 from the same institute, all in industrial engineering. He has worked for Chrysler Corporation (Chrysler Technology Center), USA, from mid-1995 through early 
1997 as a CAD/CAM software system developer. He joined Yeungnam University in early 1997. His research interests are in surface modeling, multiaxis NC machining, and off-line programming.

Byoung $\mathrm{K}$. Choi is a professor of industrial engineering. He received a BS from Seoul National University, Korea, in 1973, an MS from the Korea Advanced Institute of Science and Technology in 1975, and a $\mathrm{PhD}$ from Purdue University, USA in 1982, all in industrial engineering. He joined KAIST in early 1983, and spent a year at Purdue in 1988, and half a year at UNH (University of New Hampshire) in 1996 through early 1997, as a visiting professor. His current research interests include surface modeling, CAD/CAM, CAPP, and discrete-event system modeling and simulation. 